\title{
Género Trichomyia Haliday, 1839 (Diptera: Psychodidae) asociado con Ficus Linn. (Moraceae) en la Amazonia Colombiana
}

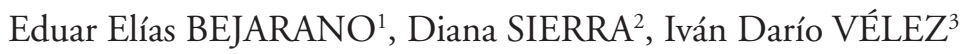

\section{RESUMEN}

Se registra por primera vez en la Amazonia colombiana la presencia del género Trichomyia Haliday, 1839, con especímenes hembras recolectados en el municipio de Leticia, capital del departamento de Amazonas. Los psicódidos se capturaron usando trampas de papel embebido con aceite de ricino, situadas durante siete días, en el tronco y las raíces tabulares de árboles de Ficus Linn. en las orillas de un pantano. Se presenta una breve descripción taxonómica de los psicódidos recolectados y se discute su asociación a este género arbóreo.

Palabras-clave: Psychodidae, Trichomyia, Ficus, Amazonia, Colombia.

\section{Genus Trichomyia Haliday, 1839 (Diptera: Psychodidae) associated with Ficus Linn. (Moraceae) in the Colombian Amazon}

\section{ABSTRACT}

The genus Trichomyia Haliday, 1839, is recorded for the first time in the Colombian Amazon, based on females collected in the municipality of Leticia, capital of the department of Amazonas. Psychodid flies were captured on sheets of paper impregnated with castor oil. The sticky traps were placed for seven days on trunks and tabular roots of Ficus Linn. trees at the edges of marsh habitats. A short taxonomic description of the collected specimens is included and the association between Trichomyia and this tree genus is discussed

KEY WORDS: Psychodidae, Trichomyia, Ficus, Amazon, Colombia.

${ }_{1}$ Grupo de Investigaciones Biomédicas, Universidad de Sucre, Cra. 14 No. 16 B-32, A.A. 406, Sincelejo, Sucre. e-mail: eduarelias@yahoo.com

2 Department of Microbiology, Immunology and Pathology, Colorado State University, Central receiving 200 W. Lake St. Campus Delivery 1619, C0 80523. e-mail: dsierra68@yahoo. com

3 Programa de Estudio y Control de Enfermedades Tropicales - PECET, Universidad de Antioquia, Calle 62 No. 52-59, A.A. 1226, Medellín, Antioquia. e-mail: id_velez@yahoo.com 
Los psicódidos son insectos holometábolos que desarrollan su ciclo de vida en ecosistemas acuáticos o terrestres. Aunque a nivel mundial es escaso el conocimiento de los hábitat de las especies del genero Trichomyia Haliday, 1839, los pocos trabajos realizados indican que se crían en ambientes húmedos ricos en madera en descomposición como agujeros de árboles, huecos entre raíces tabulares y troncos caídos (Duckhouse, 1978). Los adultos también han sido recolectados reposando sobre las hojas de pequeños árboles y arbustos (Duckhouse, 1972).

Con la excepción del registro de T. brevitarsa (Rapp, 1945), no existen estudios sobre este género en Colombia (Bejarano, 2006a,b), debido a que el interés se ha dirigido de manera casi exclusiva a las especies hematófagas de Lutzomyia França, 1924, que participan en la transmisión de microorganismos patógenos al humano (Sherlock, 2003). Durante una búsqueda de psicódidos en la Amazonia colombiana, se recolectaron varios ejemplares de Trichomyia sobre árboles, representando el primer registro del género en esta unidad biogeográfica del país.

Las recolecciones entomológicas se realizaron en Noviembre de 2003 en el municipio de Leticia, capital del departamento de Amazonas, Colombia, localizado a los $04^{\circ} 12^{\prime}$ de latitud sur y $69^{\circ} 56^{\prime}$ de longitud oeste, sobre el margen izquierdo del Río Amazonas. La temperatura media es de $27^{\circ} \mathrm{C}$ y la precipitación promedio anual de $3248 \mathrm{~mm}$. El territorio municipal es plano con pendientes mínimas que alcanzan los 90 metros sobre el nivel del mar y con áreas pantanosas. La zona de vida corresponde a bosque húmedo tropical (bh-T), según el sistema de clasificación de Holdridge (1967). Para la captura se usaron trampas de papel de 20 x $20 \mathrm{~cm}$ embebidas en aceite de ricino, las cuales fueron ubicadas durante siete días, en las raíces y los huecos de árboles situados a orillas de un pantano. Los psicódidos recolectados se transportaron hasta el Laboratorio de Entomología del Programa de Estudio y Control de Enfermedades Tropicales (PECET) de la Universidad de Antioquia. Posteriormente, éstos fueron lavados con etanol $96 \%$, tratados con lactofenol y montados en láminas portaobjeto usando el medio de Hoyer. La determinación genérica se realizó con la clave taxonómica de Quate (1996).

Dentro del material entomológico se identificaron cinco hembras del género Trichomyia, que fueron recolectadas sobre árboles de Ficus sp., las cuales presentaron cuatro venas radiales, con la vena $\mathrm{CuA}_{2}$ extendiéndose más allá de la furca media del ala. Los ojos son compuestos y carecen de puente interocular. Sus piezas bucales son cortas y sin desarrollo de mandíbulas, lo que las hace no aptas para ejercer la hematofagia. La antena está constituida por 16 segmentos excéntricos, con el escapo y pedicelo subesféricos. Adicionalmente, los palpos maxilares se encuentran divididos en tres segmentos, destacándose la presencia de una gran depresión circular con numerosas sensilas en el primer palpómero. Su genitalia es simple con un par de cercos grandes recubiertos por setas finas, mientras la mitad apical del apodema basal de la espermateca exhibe bordes casi rectos.

Debido a que la mayoría de especies de Trichomyia han sido descritas con base en machos, a la fecha no existen claves taxonómicas para la determinación de especie en las hembras, muchas de las cuales son desconocidas para la ciencia. No obstante, las hembras de la amazonia colombiana difieren de las de T. brevitarsa del litoral Caribe, entre otros caracteres, por que las últimas tienen los palpos divididos en cuatro segmentos, con el primer y segundo palpómero parcialmente fusionados. Siguiendo la propuesta de Bravo (2000) para el mencionado género, los ejemplares recolectados en la amazonia colombiana pertenecerían a un grupo monofilético que alberga los Trichomyiinae con los palpos maxilares divididos en tres segmentos, formando, además, un probable linaje evolutivo independiente caracterizado por la tenencia de flagelómeros excéntricos.

En el Neotrópico es bastante limitada la información sobre los potenciales hábitat y las especies vegetales asociadas a este género de insectos. Las únicas menciones previas en la literatura corresponden al hallazgo de T. pedicillata (Satchell, 1956) en el agujero de un árbol en Panamá, y a la descripción de la clase de vegetación de la localidad tipo de T. dolichopogon Alexander, Freitas y Quate, 2001, y T. riodocensis Alexander, Freitas y Quate, 2001, en Brasil (Satchell, 1956; Alexander et al., 2001). Por lo anterior, el presente trabajo constituye no sólo el primer registro del género Trichomyia en la amazonia colombiana, sino también la primera evidencia de su asociación con árboles del género Ficus.

Los psicódidos estudiados están depositados en la "Colección de Vectores y Hospedadores Intermediarios de Enfermedades Tropicales (VHET)" del PECET de la Universidad de Antioquia, en Medellín, Colombia.

\section{BIBLIOGRAFÍA CITADA}

Alexander, B.; Freitas, J.M.; Quate, L.W. 2001. Some Psychodidae (Diptera) from Atlantic forest in South-Eastern Brazil, with descriptions of Trichomyia dolichopogon sp. nov. and Trichomyia riodocensis sp. nov. Brazilian Journal of Biology, 61(3): 467474.

Bejarano, E.E. 2006a. Subfamilia Trichomyiinae (Psychodidae) en el territorio continental e insular colombiano. Acta Biológica Colombiana, 11(1): 37-41.

Bejarano, E.E. 2006b. Lista actualizada de los psicódidos (Diptera: Psychodidae) de Colombia. Folia Entomológica Mexicana, 45(1): 47-56. 
Bravo, F. 2000. Descrição de uma espécie de Trichomyia (Diptera, Psychodidae) do sudeste brasileiro, com comentários sobre a genealogia do gênero. Acta Biologica Leopoldensia, 22(2): 185192.

Duckhouse, D.A. 1972. Psychodidae (Diptera, Nematocera) of South Chile, subfamilies Sycoracinae and Trichomyiinae. Transactions of the Royal Entomological Society of London, 124(3): 231-268.

Duckhouse, D.A. 1978. Taxonomy, phylogeny and distribution of the genus Trichomyia (Diptera, Psychodidae) in Australia and New Guinea. Systematic Entomology, 3(3): 197-243.

Holdridge, L.R. 1967. Life zone ecology. Tropical Science Center, San José, Costa Rica. 206p.
Quate, L.W. 1996. Preliminary taxonomy of Costa Rican Psychodidae (Diptera), exclusive of Phlebotominae. Revista de Biología Tropical, 44(Supl.1): 1-81.

Satchell, G.H. 1956. On the genus Trichomyia Haliday (Diptera: Psychodidae), with descriptions of four new species. Proceedings of the Royal Entomological Society of London, Series B, 25: 147156.

Sherlock, I.A. 2003. Importância Médico-Veterinária. In: Rangel, E.F.; Lainson, R. (Eds). Flebotomíneos do Brasil. Editora Fiocruz, Rio do Janeiro, Brasil. p. 15-21.

Recebido em 14/02/2008

Aceito em 16/09/2008 
